\title{
ANÁLISIS IN-SILICO DE MUTACIONES PUNTUALES EN EL GEN PRKAG3 BOVINO ASOCIADAS A CALIDAD LA CÁRNICA
}

\author{
COMPUTATIONAL ANALYSIS OF PUNCTUAL MUTATIONS IN BOVINE PRKAG3 GENE \\ ASSOCIATEDTOMEAT QUALITY
}

Leal-Gutiérrez, J.* y Jiménez-Robayo, L.

Departamento de Ciencias de la Producción Animal. Facultad de Medicina Veterinaria y de Zootecnia. Universidad Nacional de Colombia, sede Bogotá. Bogotá. Colombia. *jdlealg@unal.edu.co

\section{Palabras CLAVE ADICIONALES}

AMPK. Glicógeno muscular. Potencial electrostático. Punto isoeléctrico.

\section{RESUMEN}

La molécula AMPK posee como función mantener el nivel energético de la célula muscular y la subunidad PRKAG3 regula su actividad, por lo que esta última se ha convertido en uno de los genes candidatos en estudios de asociación fenotipogenotipo en animales de granja. Se realizó una búsqueda sobre el efecto in-silico de las mutaciones recogidas en bases de datos que se ubican en regiones exónicas del gen PRKAG3 bovino, con el fin de determinar los principales SNPS a evaluar en estudios de asociación, según la modificación de algunos parámetros físico-químicos del ARNm o proteína. Los polimorfismos 1796C y $2338 \mathrm{~T}$ en el ARNm y el 389T en la secuencia proteica del PRKAG3 bovino son los SNPs con más posibilidades de contribuir a la variación fenotípica encontrada en la carne de bovino en la bibliografía consultada. Adicionalmente, se estableció la mutación 200Q (RN-) del cerdo como causante de varias modificaciones en parámetros químicos de la proteína in-silico, lo que podría dar respuesta al marcado efecto que posee sobre la calidad cárnica en esta especie.

\section{SUMMARY}

PRKAG3 protein is one of the three components of AMPK and it has a regulatory function in this molecule. This gen had been associated with several quality traits of porcine meat. Computational analysis of mutations in exonic region was established using SNP (single nucleotide polymorphism) reported in datebases. The aim

\section{AdDitionAL KEYWORDS}

AMPK. Muscle glycogen. Electrostatic potential. Isoelectric point.

was highlight the mutations that can change some physicochemical traits in RNAm and/or protein. Polymorphism 1796C and 2338T in ARNm and 389T in protein of bovine PRKAG3 were the most important because they theoretically can modify the normal working of these molecules. Additionally, in-silico, the porcine polymorphism 200Q (RN-, strongly associated to meat quality) modifies several features of RNAm and its protein.

\section{INTRODUCCIÓN}

Una de las moléculas más importantes en estudios de asociación entre polimorfismos genéticos y atributos de calidad cárnica en cerdo es la proteína PRKAG3, que es una de las moléculas constituyentes de la AMPK. La proteína AMP kinasa activada (AMPK) se considera un sensor de la energía celular; siendo sensible al incremento de la concentración de AMP en respuesta a una disminución en el nivel energético de la célula o a estrés, así como a un incremento en la tasa AMP:ATP (Grahame et al., 2005). La proteína PRKAG3 es la encargada de la activación de la molécula AMPK (Xiao et al., 2011), encontrándose así involucrada en su regulación en el músculo esquelético y por tanto en el metabolismo del glicógeno. Los estudios de asociación de polimorfismos del gen PRKAG3 


\section{LEAL-GUTIÉRREZY JIMÉNEZ-ROBAYO}

y la calidad cárnica, se basan en alteraciones en la deposición del glicógeno en el músculo antes del sacrificio del animal y su conversión en ácido láctico durante el período post-mortem, influyendo directamente sobre varios atributos de calidad de la carne (Ouali et al., 2006).

El gen de la proteína PRKAG3 presenta una alta variabilidad en diferentes razas de bovinos, siendo considerado un gen candidato asociado a alteraciones fisiológicas con influencia sobre la calidad del producto final. En el caso del cerdo son más reducidas las mutaciones puntuales depositadas en bancos de datos (www.ncbi.nlm.nih.gov), pero según múltiples estudios de asociación en cerdos se han podido identificar varias mutaciones, la rn* (199I-200R) y la RN- (199V-200Q) que poseen asociación con variaciones en calidad y el denominado como alelo silvestre, rn+ (199V-200R) (Laville et al., 2009; Lahucky et al., 1997; Otto et al., 2007). De lo anterior se establece la existencia de un efecto fenotípico preponderante, causado por las mutaciones que van a modificar la constitución del ARNm y/o la proteína y por lo tanto sus funciones biológicas.

Según Duan et al. (2003), en los estudios de asociación con genes candidatos es ideal emplear SNPs y otras mutaciones funcionales, donde la identificación de sustituciones nucleotidicas puede tener implicaciones importantes para el diseño e interpretación de estudios de asociación con características fenotípicas, como es el caso de la calidad cárnica. Por lo tanto, el objetivo de esta investigación es establecer alteraciones en parámetros físico-químicos, generadas por mutaciones puntuales reportadas en el gen PRKAG3 bovino a nivel del ARNm y proteico mediante análisis computacional y establecer cuáles son los SNPs con mayor capacidad de alterar funcionalmente estas moléculas y por lo tanto a nivel fenotípico en bovino, empleando los alelos rn*, RN-y $\mathrm{rn}+$ del cerdo como polimorfismos guía en este proceso dado sus efectos fenotípicos en el cerdo.

\section{METODOLOGÍA}

\section{SeCUENCIASY SNPSEMPLEAdOS}

Las secuencias de ARNm empleadas fueron: NM_001030302.2y NM_214077.1 (bovino y porcino respectivamente); para proteína: DAA32407.1 y AAP12533.1 (bovina y porcina respectivamente), en este articulo, estas secuencias son denominadas como silvestres. Se utilizaron los SNPs de las regiones exónicas del gen PRKAG3 bovino (tabla 1) y los tres alelos $\left(\mathrm{rn}+, \mathrm{rn}^{*} \mathrm{y}\right.$ $\mathrm{RN}-$ ) descritos en estudios de asociación genotipo-fenotipo en cerdo. En las secuencias mencionadas anteriormente se realizó la sustitución manual para la formación de cada una de las moléculas de ARNm y proteína (dado el caso) mutadas (el alelo rn+ es denominado como la secuencia silvestre del cerdo).

\section{Modelamiento del ARNM}

La estructura secundaria más probablemente adoptada por las secuencias del ARNm y sus valor de Energía Mínima Libre $(\Delta G)$ se determinaron mediante el software MFold (Zuker, 2003) a temperatura fisiológica.

\section{MODELAMIENTOPROTÉICO}

La estructura secundaria de las secuencias proteicas se obtuvieron mediante el servidor Quick2d (toolkit.tuebingen.mpg. de/quick2_d) y las regiones desordenadas con DISOPRED (Ward et al., 2004). Adicionalmente se detectaron los dominios constituyentes de la proteína mediante el servidor CD-Search (Marchler y Bryant, 2004) del NCBI. El modelamiento por homología fue empleado para la determinación de la estructura terciaria (PRKAG3), usando el servidor SwissModel y el Software Deep Viewer (Guex y Peitsch, 1997, www.expasy.org/spdbv/), efectuando la minimización energética de cada proteína 
mediante Gromos96. La proteína PRKAG3 bovina modelada, fue incluida en la estructura cuaternaria adoptada por la molécula AMPK depositada en PDB, la estructura cristal 2Y94 (Xiao et al., 2011) mediante la opción Magic Fit del Software Deep Viewer (Guex y Peitsch, 1997; http://www.expasy. org/spdbv/). Los parámetros físico-químicos se determinaron mediante el servidor Protparam (www.expasy.org/spdbv/) y el Software Deep Viewer (Guex y Peitsch, 1997; http://www.expasy.org/spdbv/).

\section{RESULTADOS}

En la figura 1 se presenta la estructura y tamaño del gen, el ARNm y la proteína del PRKAG3 bovino. En bases de datos (NCBI) existen reportados un total de 111 SNPs, de los cuales aproximadamente 31 modifican un nucleótido de la región exónica (tabla I) alterando la secuencia del ARNm. De estas, 10 mutaciones hacen parte de los exones del gen PRKAG3 que van a codificar la proteína y cuatro de ellas son missense (tabla I).

\section{ModelosdeARNm}

En la figura 2 se presenta la estructura secundaria adoptada por el ARNm silvestre del PRKAG3 bovino y las cinco modificaciones estructurales más importantes producidas por las mutaciones puntuales evaluadas. Para las secuencias del ARNm del gen PRKAG3 porcino silvestre (codones GTCCGA en la posición 745 del ARNm) y la configuración adquirida por la mutación

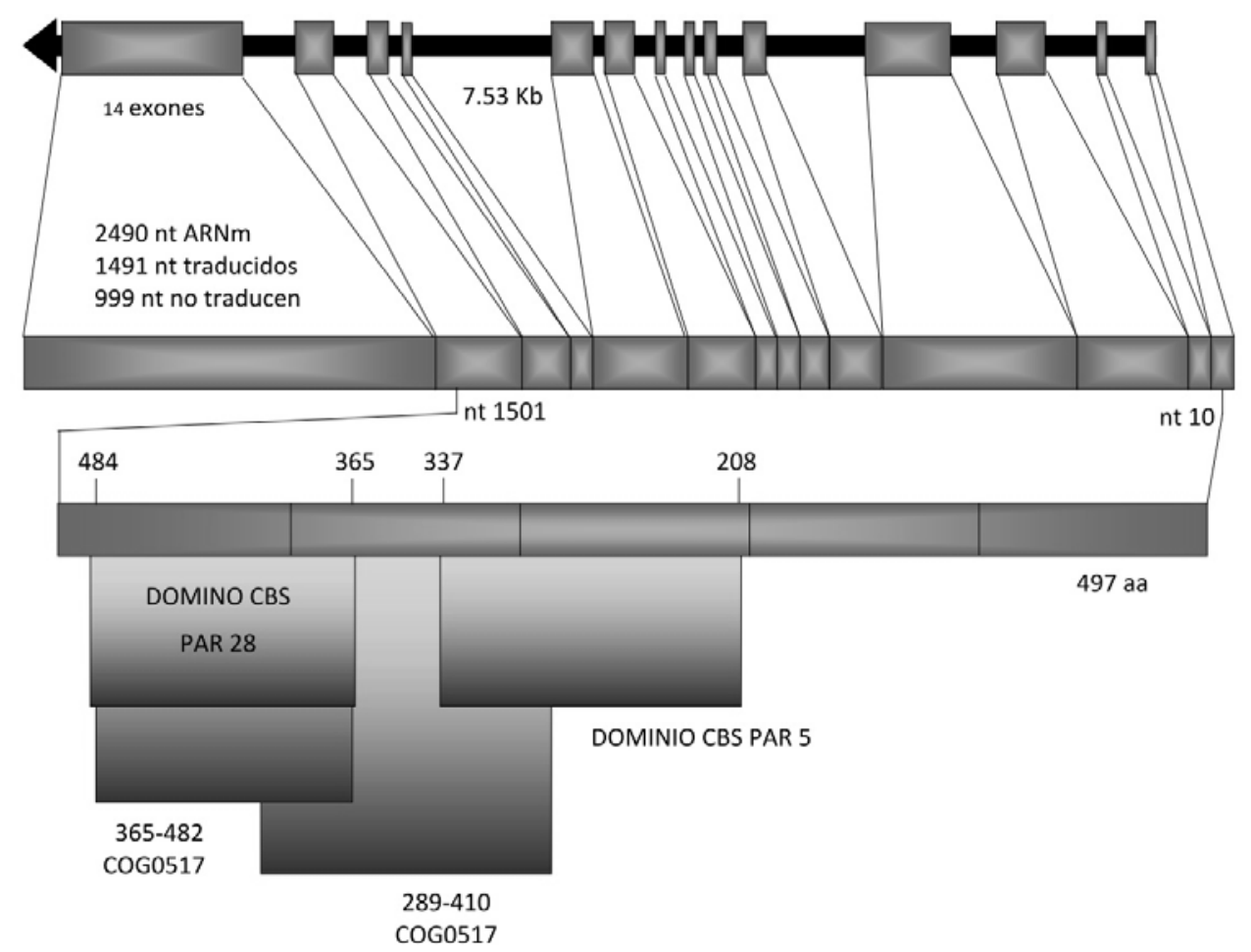

Figura 1. Estructura del gen PRKAG3 bovino, su ARNm y proteína. Se presentan los dominios predichos mediante CD-Search. (Structure of bovine PRKAG3 gen, their ARNm and protein. Predicted domains with CD-Search are shown). 
RN- (codones GTCCAA), existen leves diferencias, mientras que el alelo $\mathrm{rn}^{*}$ (codones ATCCGA) no alteró esta estructura. A nivel de estabilidad molecular, el valor de energía libre del ARNm en el alelo silvestre del PRKAG3 bovino, obtuvo un valor de -885.04 $\mathrm{kcal} / \mathrm{mol}$. En orden descendente, las mutaciones que desestabilizan en mayor medida a esta molécula son, el SNP 16, 17 y 15 con valores de $\Delta \mathrm{G}$ de $-871,60 ;-876,48$ y -879,91 $\mathrm{kcal} / \mathrm{mol}$ respectivamente y el SNP con el mayor carácter estabilizante, fue el 28 con 899,17 kcal/mol. Los modelos del ARNm de los tres alelos del cerdo presentaron un valor en $\Delta \mathrm{G}$ de $-821,86 ;-820,03$ y $-821,86$ $\mathrm{kcal} / \mathrm{mol}$ para el alelo silvestre, RN- y rn* respectivamente.

\section{MODELOPROTEICO}

En la figura 1 se presentan los dominios

Tabla I. Polimorfismos del PRKAG3 bovino empleados. (Polymorphism of bovine PRKAG3 used).

\begin{tabular}{|c|c|c|c|c|c|c|c|}
\hline $\begin{array}{l}\text { Código de } \\
\text { accesión }\end{array}$ & $\begin{array}{l}\text { ARNm } \\
\text { Posición }\end{array}$ & Silvestre & Cambio & $\begin{array}{l}\text { Proteína } \\
\text { Posición }\end{array}$ & Silvestre & Cambio & Secuencia \\
\hline s109995479 & 389 & $G$ & $\mathrm{~T}$ & 127 & $A$ & $\mathrm{~S}$ & 1 \\
\hline rs111024758 & 468 & G & $\mathrm{C}$ & 153 & W & $\mathrm{S}$ & 2 \\
\hline rs43316209 & 472 & $A$ & $\mathrm{C}$ & 154 & G & G & 3 \\
\hline rs43316214 & 742 & $\mathrm{~T}$ & $A$ & 244 & $\mathrm{~F}$ & $\mathrm{~L}$ & 4 \\
\hline rs110730865 & 793 & $\mathrm{C}$ & $\mathrm{T}$ & 261 & $Y$ & $Y$ & 5 \\
\hline rs109510654 & 844 & $\mathrm{C}$ & G & 278 & $\mathrm{~T}$ & $\mathrm{~T}$ & 6 \\
\hline rs134238119 & 945 & $\mathrm{~T}$ & $\mathrm{C}$ & 312 & I & $\mathrm{T}$ & 7 \\
\hline rs110146930 & 1432 & $\mathrm{C}$ & $\mathrm{T}$ & 474 & G & G & 8 \\
\hline rs109437532 & 1435 & G & $A$ & 475 & $\mathrm{~V}$ & V & 9 \\
\hline rs111024144 & 1495 & $\mathrm{C}$ & $\mathrm{T}$ & 495 & $\mathrm{~L}$ & $\mathrm{~L}$ & 10 \\
\hline rs109665777 & 1512 & G & $A$ & NA & NA & NA & 11 \\
\hline rs43315207 & 1527 & G & $A$ & NA & NA & NA & 12 \\
\hline rs110658325 & 1601 & A & G & NA & NA & NA & 13 \\
\hline rs43315203 & 1640 & $\mathrm{~T}$ & $\mathrm{C}$ & NA & NA & NA & 14 \\
\hline rs43315202 & 1732 & $\mathrm{C}$ & $\mathrm{T}$ & NA & NA & NA & 15 \\
\hline rs43315201 & 1796 & $\mathrm{~T}$ & $\mathrm{C}$ & NA & NA & NA & 16 \\
\hline rs43315200 & 1804 & $\mathrm{~T}$ & $\mathrm{C}$ & NA & NA & NA & 17 \\
\hline rs43315199 & 1897 & $A$ & G & NA & NA & NA & 18 \\
\hline rs43315198 & 1991 & $\mathrm{C}$ & $\mathrm{T}$ & NA & NA & NA & 19 \\
\hline rs43315197 & 2024 & $\mathrm{~T}$ & $\mathrm{C}$ & NA & NA & NA & 20 \\
\hline rs110316264 & 2070 & $\mathrm{C}$ & $\mathrm{T}$ & NA & NA & NA & 21 \\
\hline rs43315196 & 2123 & $\mathrm{C}$ & G & NA & NA & NA & 22 \\
\hline rs43315195 & 2197 & $A$ & G & NA & NA & NA & 23 \\
\hline rs43315194 & 2213 & G & $\mathrm{C}$ & NA & NA & NA & 24 \\
\hline rs43315193 & 2257 & $\mathrm{C}$ & $\mathrm{T}$ & NA & NA & NA & 25 \\
\hline rs43315192 & 2265 & $\mathrm{~T}$ & $\mathrm{C}$ & NA & NA & NA & 26 \\
\hline rs43315191 & 2302 & G & A & NA & NA & NA & 27 \\
\hline rs43315190 & 2338 & $\mathrm{C}$ & $\mathrm{T}$ & NA & NA & NA & 28 \\
\hline rs43315189 & 2359 & G & $A$ & NA & NA & NA & 29 \\
\hline rs43315188 & 2386 & $\mathrm{C}$ & $\mathrm{T}$ & NA & NA & NA & 30 \\
\hline rs43315187 & 2388 & $\mathrm{~T}$ & $\mathrm{C}$ & NA & NA & NA & 31 \\
\hline
\end{tabular}

Ubicación en la secuencia del ARNm y la proteína. NA= polimorfismo que no afecta la secuencia de la proteína. 


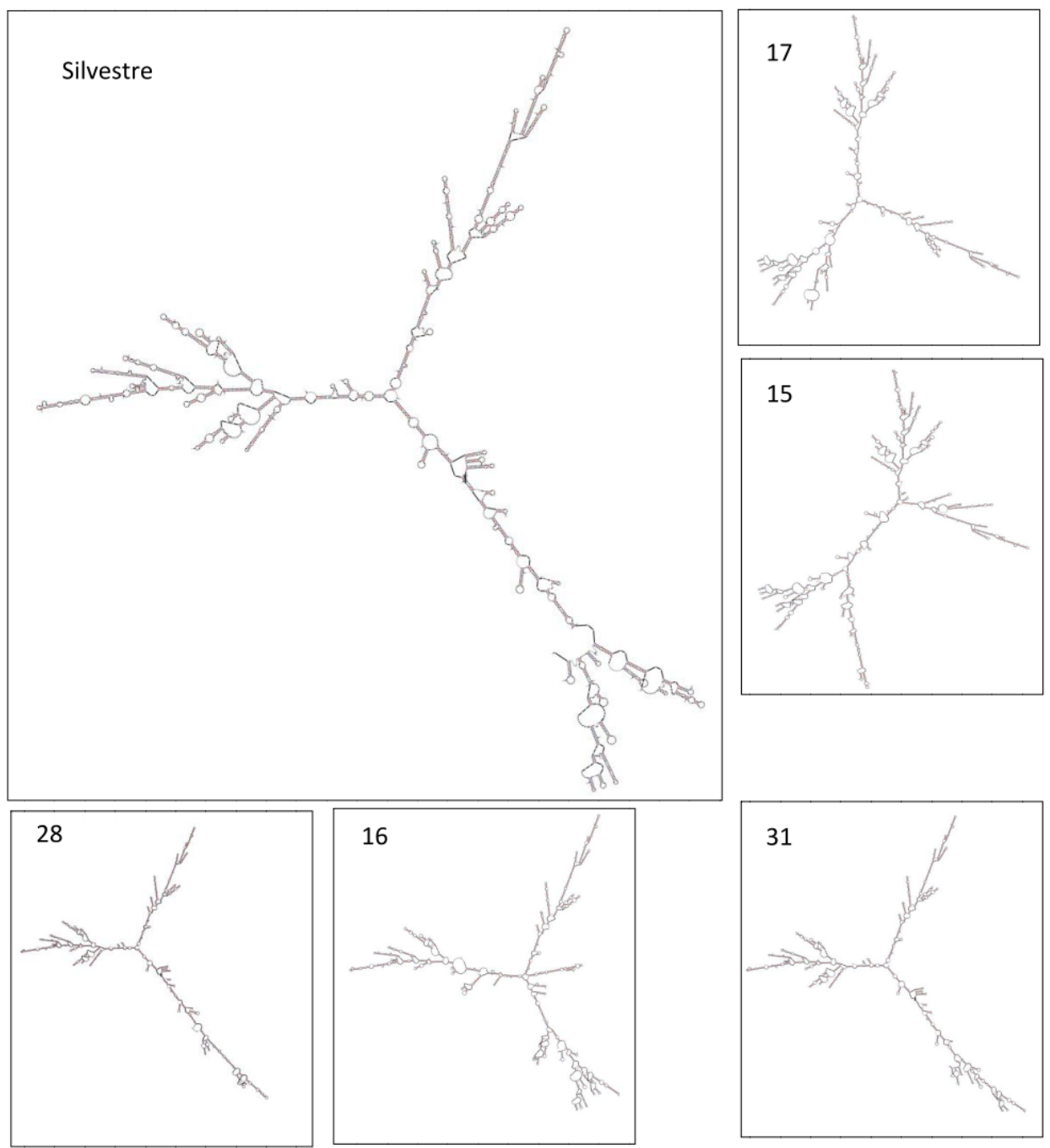

Figura 2. Polimorfismos conformacional del ARNm del bovino. Se presentan las cinco mutaciones más importantes según modificación de la estructura bidimensional.(Conformational polymorphism ob bovine RNAm. The most important mutations depending of bidimensional structure are shown).

constituyentes de la proteína PRKAG3 bovina. Para el modelamiento en 3D de la molécula, el servidor Swiss Model empleó el templete 2v8qE (posee como ligando dos moléculas de AMP) con una identidad del $64,78 \%$, logrando modelar desde el ami- noácido 188 hasta el 488 de la secuencia problema, por lo que no todas las cuatro mutaciones que modifican un aminoácido en la cadena proteica pudieron ser modeladas, únicamente las mutaciones 4 (aa 244) y 7 (aa 312) del bovino. Las mutaciones rn* 
y RN- modifican respectivamente el aminoácido 199 y 200, por lo que si se lograron modelar. En la figura 3 se presenta la estructura cuaternaria del AMPK con el modelo obtenido para la proteína PRKAG3 bovina y se ubican las posiciones 244, 312 en la proteína bovina y 199 y 200 en la proteína del cerdo sujetas a cambio según el SNP eva-
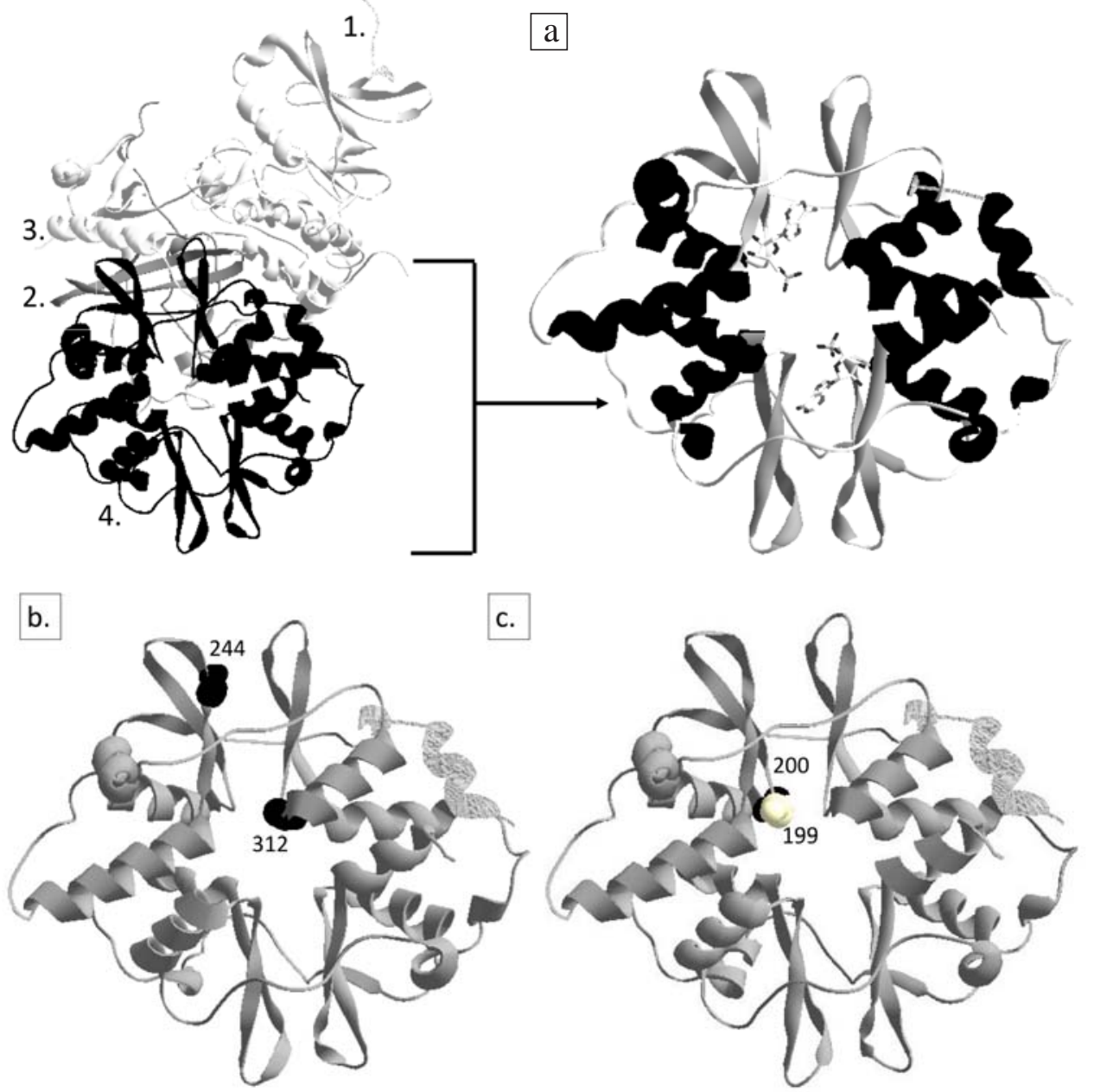

Figura 3. Molécula activa del AMPK. a. Subunidades 1 y 3 corresponden al PRKAA1, subunidad 2 al PRKAB2 y 4 es el modelo del PRKAG3 bovino obtenido. Se detalla el PRKAG3 señalando la estructura secundaria adquirida; en gris oscuro hojas- $\beta$, en negro $\alpha$-hélices. b. Ubicación de los aminoácidos 244 y 312 en la proteína PRKAG3 del bovino. c. Ubicación de los aminoácidos 199 y 200 involucrados en cambios mutaciones en el cerdo. (AMPK active molecule. a. Subunities 1 and 3 correspond to PRKAA1; subunity 2 correspond to PRKAB2, and 4 is the obtained bovine PRKG3 model; latter shows secondary structure. $\beta$-sheets in black, $\alpha$-helix in gray. b. Location of 244 and 344 aminoacids in PRKAG3 bovine protein. c. Location of 199 and 200 amino acids involved in missense mutations in pigs). 
luado. La figura 4 detalla el sitio activo (unión de las dos moléculas de AMP intercambiables, (Xiao et al., 2011) de la proteína PRKAG3 en área de contacto y los puentes de Hidrógeno entre las estas moléculas y los aminoácidos involucrados en su fijación.

La figura 5 ilustra la distribución del campo electromagnético de las secuencias silvestres bovina y porcina y su mutación $\mathrm{RN}-$, como la única que altera notablemente este parámetro.

\section{DISCUSIÓN}

Según la base de datos consultada, es importante destacar la alta variabilidad del gen PRKAG3 bovino, que es superior al total de SNPs descritos para el cerdo (13, no correspondiendo ninguna a las dos mutaciones empleadas para el análisis en este artículo), lo que induce a considerar a este gen como un potencial candidato en estudios de asociación fenotipo-genotipo, en el área de calidad cárnica en bovino dada su función biológica y el rol que desempeña durante el post-mortem en la conversión del músculo a carne. Otras investigaciones destacan la alta variabilidad de este gen, entre los que se citan a Yu et al. (2005) quienes identificaron tres mutaciones funcionales y un splicing alternativo en el exón 2 y Roux et al. (2006) con 32 SNPs adicionales, cinco de los cuales son funcionales.

Barnes et al. (2005) lograron establecer una mutación en el gen PRKAG3 humano (R225Q), que altera la deposición in vivo a nivel muscular del glicógeno, dada una pérdida de la función de la proteína al eliminar la regulación realizada por la relación AMP:ATP, incrementando la actividad basal del AMPK. El principio anterior, es también señalado por otros investigadores (por ejemplo Enfält et al., 2009 y Lindahl et al., 2004a) como el responsable de alterar la calidad cárnica en el cerdo (asociado al alelo RN-) al existir una mayor deposición de glicógeno ante-mortem que conduce a una caída de $\mathrm{pH}$ más acelerada en el post-morten, dada su conversión a ácido láctico. Por otro lado, Schefûer et al. (2011) indicaron que el cambio en la deposición de glicógeno es solo una de las modificaciones que presentan los animales con la variante $\mathrm{RN}$ - respecto a los que expresan la proteína normal, por lo que sugieren la necesidad de establecer diferencias tanto en las propiedades como en la cantidad y la actividad de la enzima, el tipo de fibra muscular y su estatus energético.

\section{VARIACIONES ENELARNM}

La importancia del ARNm no únicamente se basa en la información contenida en él, que aparece formando un texto que se lee linealmente ó la denominada información digital, sino también a la concepción análoga del ARNm explicada por Andrade (2011). La distinción análogo-digital es crucial si se quiere entender los fundamentos fisicoquímicos de la información genética, puesto que la primera hace posible la aparición de la segunda, donde la actividad biológica depende de las enzimas, cuya estructura tridimensional les permite reconocer sus sustratos y por tanto actuar en consecuencia (Andrade, 2011). La funcionalidad y el mantenimiento de la actividad metabólica requieren de enzimas que oscilan entre configuraciones estructurales muy precisas y la importancia de los reconocimientos específicos entre aminoácidos, enzimas aminoacil-RNA-sintetasas y los respectivos RNA de transferencia pone de relieve la importancia de la información analógica que posibilita la existencia y el funcionamiento de la digital (Andrade, 2011). Por lo tanto la estructura tridimensional (o en este caso la aproximación mediante la estructura secundaria adoptada por el ARNm) y su modificación por mutaciones puntuales puede ser una fuente de variación fenotípica, al alterar por ejemplo, la tasa de traducción (Johnson et al., 2011). Relacionado con lo anterior, Duan et al. (2003) establecen que aunque una mutación 
(C957T) del gen del Receptor de la Dopamina D2 (DRD2) humano es sinónima, modifica la estructura análoga adoptada por el ARNm (evaluando su estructura secundaria), disminuyendo su estabilidad (vida media del ARNm silvestre de 8 horas a 4 horas en el mutante 957T) y por lo tanto su eficiencia en traducción in vitro hasta en un 50 \%, cambiando dramáticamente la expresión del DRD2.

Para el caso del ARNm del gen PRKAG3 bovino, cinco mutaciones modifican noto- riamente la estructura predicha (figura 2), por lo que potencialmente pueden afectar la expresión del gen. Adicionalmente la estabilidad de esta molécula es claramente afectada por cuatro de ellas (valores de $\Delta \mathrm{G}$ ), donde la mutación 16 (1796C) aunque no modifica un aminoácido en la proteína, disminuye la estabilidad del modelo de ARNm. Teóricamentela mutación con el mayor efecto estabilizante de la molécula de ARNm corresponde a la número 28 (2338T), la cual tampoco forma parte de un codón de traduc-

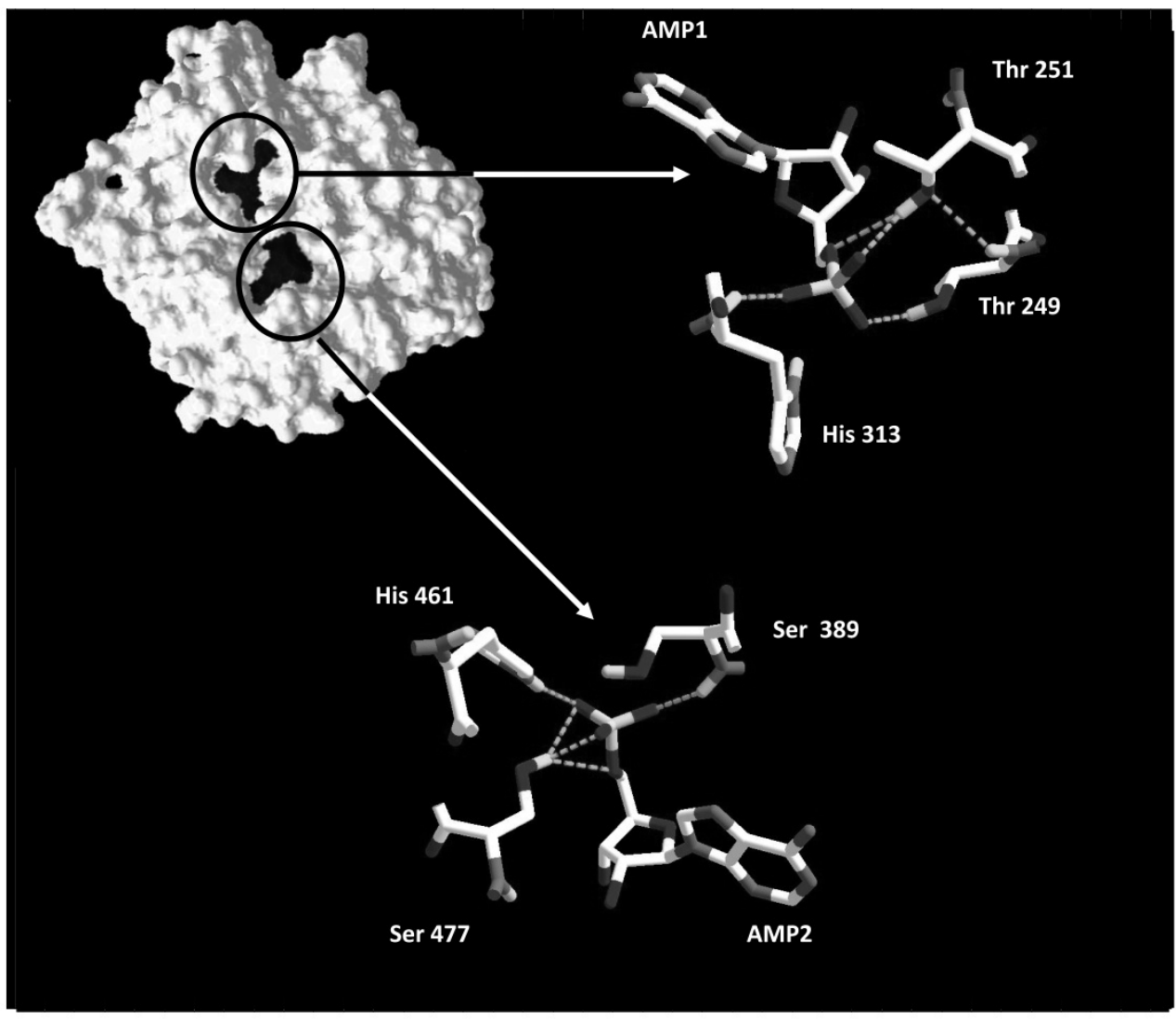

Figura 4. Sitio activo del PRKAG3 bovino. Área de contacto de la superficie del PRKAG3 y la superficie de las dos moléculas de AMP intercambiables, se detallan los aminoácidos involucrados en la fijación de estas últimas mediante enlaces de Hidrógeno. (Active site of bovine PRKAG3. Contact area between PRKAG3 and interchangeable AMP molecules; the amino acids involved in their fixation via hidrogen bonds are detailed).

Archivos de zootecnia vol. 63, núm. 241, p. 128. 
ción. Lo anterior se basa en el hecho de que algunas mutaciones que no afectan a la proteína pero si al ARNm se pueden asociar a variaciones en el fenotipo, como lo mencionan Johnson et al. (2011), al establecer que los polimorfismos conformacionales del ARNm producidos por SNPs funcionales en genes ya asociados con fenotipos humanos, contribuyen sustancialmente a su variabilidad fenotípica.

\section{LAPROTEÍNAPRKAG3 Y LAS ALTERACIO- NES POR SNPS}

Es interesante resaltar la predicción de varias regiones desordenadas en la secuencia lineal de la proteína hasta el aminoácido 185, concordando con el segmento que se logró modelar a nivel terciario, lo que sugiere que este fragmento no forma parte de la molécula activa, estando ésta comprendida entre los aminoácidos 188 y 488, en donde se distribuyen los dominios CBS Par 28 y CBS Par 5, que generalmente forman un par de dominios CBS intramolecular, como un sitio potencial de fijación a un ligando, por lo que desempeñan una función generalmente reguladora (información suministrada por CD-Search, Marchler y Bryant, 2004), lo que está en concordancia con la estructura terciaria obtenida, dada una configuración globular (figura 3) y en su interior una región en donde se ligan dos moléculas de AMP intercambiables (Xiao et al., 2011).

Las cuatro mutaciones del bovino que modifican un aminoácido en la secuencia proteica alteran su peso molecular levemente (datos no mostrados). Pero uno de los parámetros que en mayor medida es modificado por las mutaciones missense, es el valor del punto isoeléctrico (PI). Bee et al. (2007) establecieron que el pH en las primeras horas post-mortem se relaciona con la degradación de proteínas citoesqueléticas, ocurriendo más rápido en carcasas que presentaban $\mathrm{pH}$ inferiores a 5,7 a las 3 horas post-mortem. De tal modo que la caída del pH puede influir en la disrupción proteica, al permitir la activación de enzimas proteolíticas como el sistema CalpainaCalpastatina, pero en este mismo sentido, la disminución gradual del $\mathrm{pH}$ en la carne generara la inactivación de estos mismos sistemas enzimaticos. Si un mutación permite que enzimas como el AMPK sea inactivado (por ejemplo incrementando el valor del PI en la proteína reguladora PRKAG3) los procesos mediados por esta enzima serán limitados en el post-mortem y viceversa, si la mutación le confiere una actividad más prolongada al disminuir su valor de PI. Por lo anterior, en el caso del bovino únicamente una de las cuatro mutaciones genera una modificación en este parámetro, la mutación 1 (389T) pasando su valor de PI de 5,20 a 5,26 , haciendo que la enzima presente una menor actividad en el post-mortem y según su función biológica, una menor conversión de glicógeno a ácido láctico, teniendo como resultado un mayor $\mathrm{pH}$ final en la carne o un descenso más gradual.

Un hallazgo que puede sustentar la afirmación anterior, es revelado por el valor de PI conferido por la mutación RN- a la proteína del cerdo, pasando de 5,24 a 5,18, es decir esta molécula podría conferirle una mayor actividad a la molécula AMPK, traduciéndose en un valor de $\mathrm{pH}$ final mucho más bajo de lo normal, algo totalmente en concordancia con la calidad cárnica atribuida a animales con esta mutación. Así mismo, Lindahl et al. (2004b), empleando el músculo Longissimus dorsi de cerdos, describieron un efecto negativo del alelo $\mathrm{RN}$ - sobre el pH final en carne cruda, al ser comparado con los alelos rn+ y rn*, estableciendo la siguiente jerarquía para la característica pH final: genotipos $\mathrm{RN}-/-<\mathrm{rn}+/$ $\mathrm{rn}+<\mathrm{rn}+/ \mathrm{rn}^{*}=\mathrm{rn} * / \mathrm{rn}^{*}$, concluyendo que los cerdos cuyo genotipo poseía al menos una copia del alelo RN- presentaba un $\mathrm{pH}$ más bajo en su carne. Pero lo anterior no es aplicable para el alelo rn*, ya que este SNP no modifica el valor de PI, aunque según Lindahl et al. (2004) si presentan una diferencia significativa en el valor de $\mathrm{pH}$ 
final de la carne respecto al alelo rn+. Cabe resaltar la gran complejidad de eventos a nivel genético que conducen a la conversión del músculo a carne (genes que intervienen es este proceso, efecto de sus mutaciones e interacciones entre ellos).

De las figuras $\mathbf{3}$ y $\mathbf{4}$ se resalta la ubicación de las dos moléculas de AMP en el interior de la proteína PRKAG3 (según Xiao et al., 2011 existe una tercer molécula de AMP fijada, pero no es intercambiable). En la figura 4 se ilustra la fijación de ambas moléculas de AMP a la proteína mediante enlaces de hidrógeno (AMP1 mediante cuatro y AMP2 por cinco enlaces). Ninguna de las mutaciones evaluadas modifica estos
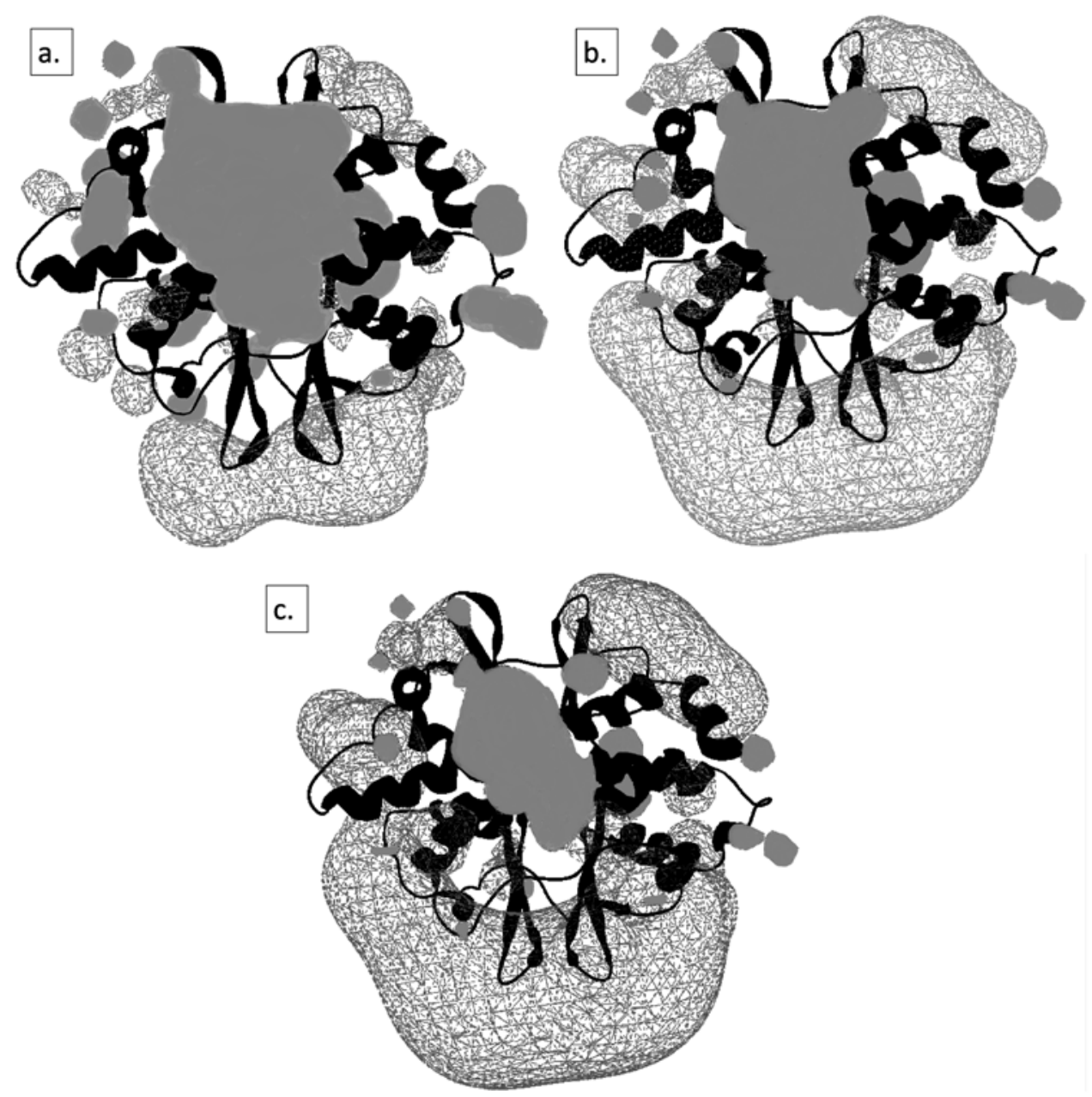

Figura 5. Potencial electroestático de a. proteína bovina silvestre, b. proteína silvestre porcina y c. proteína mutada $R N$-; potencial positivo en gris, potencial negativo en líneas punteadas y la proteína en negro. (Electrostatic potential of. a. wild bovine protein; b. wild pig protein, and c. mutated protein RN-; positive potential in gray, negative potential in doted lines and protein in black). 


\section{ANÁLISIS IN-SILICO DE MUTACIONES DEL GEN PRKAG3 BOVINO}

enlaces de Hidrógeno. En el caso del cerdo la mutación RN- logra modificar el potencial electroestático de la proteína (figura 5) incrementando el potencial negativo de la molécula y disminuyendo el potencial positivo existente al interior (lugar de fijación a AMP), lo que podría modificar su asociación a su ligando y/o su ensamblaje a la molécula final de AMPK. Ninguna de las dos mutaciones modeladas modificaron el potencial electrostático del PRKAG3 bovino y en el caso del porcino, la mutación rn* mantiene la misma distribución que el alelo silvestre, por lo que no explicaría las diferencias fenotípicas que le confiere a la carne.

\section{CONCLUSIONES}

Las mutaciones del gen PRKAG3 bovino con un mayor potencial de causar una

\section{BIBLIOGRAFÍA}

Andrade, E. 2011. La dualidad análogo digital de la información se ejemplifica en el estudio de las moléculas de RNA. Acta Biol Colomb, 16: 1542.

Barnes, B.; Glund, S.; Chau Long, Y.; Hjälm, G.; Andersson, L. and Zierath, J. 2005. 5'-AMPactivated protein kinase regulates skeletal muscle glycogen content and ergogenics. FASEB J, 19: 773-779.

Bee, G.; Anderson, A.; Lonergan, S. and Lonergan, E. 2007. Rate and extent of $\mathrm{pH}$ decline affect proteolysis of cytoskeletal proteins and waterholding capacity in pork. Meat Sci, 76: 359-365.

Duan, J.; Wainwright, M.; Comeron, J.; Saitou, N.; Sanders, A.; Gelernter, J. and Gejman, P. 2003. Synonymous mutations in the human dopamine receptor D2 (DRD2) affect mRNA stability and synthesis of the receptor. Hum Mol Genet, 12: 205-216.

Enfält, A.; von Seth, G.; Josell, A.; Lindahl, G.; Hedebro-Velander, I.; Braunschweig, M.; Andersson, L. and Lundström, K. 2006. Effects of a second mutant allele (V199I) at the PRKAG3 (RN) locus on carcass composition in pigs. Livest Sci, 99: 131-139.

Grahame, D. 2005. New roles for the LKB1 AMPK variación fenotípica en los parámetros de calidad cárnica se puede dividir en dos grupos según los resultados obtenidos, el primero, como aquellos que pueden alterar la estructura conformacional del ARNm y modificar su energía mínima libre, alterando su estabilidad y el segundo como aquellas que pueden afectar algún parámetro físicoquímico de la proteína funcional. En el primer grupo se pueden ubicar el polimorfismo 16 (1796C, como desestabilizante) y el 28 (2338T como estabilizante) y en el segundo grupo, la mutación 1 (389T) dado su efecto sobre el PI de la molécula, posiblemente asociado a una mejor calidad cárnica. De igual manera la mutación RN- del cerdo modifica teóricamente varios parámetros de la proteína (menor PI y alteración del potencial electrostático molecular), lo que puede dar explicación a su efecto biológico.

pathway. Curr Opin Cell Biol, 17: 167-173.

Guex, N. and Peitsch, M. 1997. SWISS-MODEL and the Swiss-PdbViewer: An environment for comparative protein modeling. Electrophoresis, 18: 2714-2723.

Johnson, A.; Trumbower, H. and Sadee, W. 2011. RNA structures affected by single nucleotide polymorphisms in transcribed regions of the human genome. WebmedCentral Bioinformatics, 2: WMC001600. http://www. webmedcentral.com/wmcpdf/Article_ WMC001600.pdf (22/12/2012).

Lahucky, R.; Christian, L.; Kovaq, L.; Staldert, K. and Bauerova, M. 1997. Meat quality assessed ante- and post mortem by different ryanodine receptor gene status of pigs. Meat Sci, 41: 211285.

Laville, E.; Sayd, T.; Terlouw, C.; Blinet, S.; Pinguet, J.; Fillaut, M.; Glenisson, J. and Cherel, P. 2009. Differences in pig muscle proteome according to HAL genotype: Implications for meat quality. J Agric Food Chem, 57: 4913-4923.

Lindahl, G.; Enält, A.; Von Seth, G.; Josell, A.; Hedebro-Velander, I.; Andersen, H.; Braunschweig, M.; Andersson, L. and Lundstöm, K. 2004a. A second mutant allele (V199I) at the 


\section{LEAL-GUTIÉRREZY JIMÉNEZ-ROBAYO}

PRKAG3 (RN) locus. I. Effect on technological meat quality of pork loin. Meat Sci, 66: 609-619.

Lindahl, G.; Enält, A.; Von Seth, G.; Josell, A.; Hedebro-Velander, I.; Andersen, H.; Braunschweig, M.; Andersson, L. and Lundstöm, K. 2004b. A second mutant allele (V199I) at the PRKAG3 (RN) locus. II. Effect on colour characteristics of pork loin. Meat Sci, 66: 621627.

Marchler, A. and Bryant, S. 2004. CD-Search: protein domain annotations on the fly. Nucleic Acids Res, 32 (Web Server issue): W327-331. Otto, G.; Roehe, R.; Looft, H.; Thoelking, L.; Knap, P.; Rothschild, M.; Plastow, G. and Kalm, E. 2007. Associations of DNA markers with meat quality traits in pigs with emphasis on drip loss. Meat Sci, 75: 185-195.

Ouali, A.; Herrera-Mendez, C.; Coulis, G.; Becila, S.; Boudjellal, A.; Aubry, L. and Sentandreu, M. 2006. Revisiting the conversion of muscle into meat and the underlying mechanisms. Meat Sci, 74: 44-58.

Roux, M.; Nizou, A.; Forestier, L.; Ouali, A.; Levéziel, $\mathrm{H}$. and Amarger, V. 2006. Characterization of the bovine PRKAG3 gene: structure, polymor- phism, and alternative transcripts. Mamm Genome, 17: 83-92.

Schefûer, T.; Park, S. and Gerrard, D. 2011. Lessons to learn about postmortem metabolism using the AMPK $\gamma 3$ R200Q mutation in the pig. Meat Sci, 89: 244-250.

Ward, J.; Sodhi, J.; McGuffin, L.; Buxton, B. and Jones, D. 2004. Prediction and functional analysis of native disorder in proteins from the three kingdoms of life. J Mol Biol, 337: 635-645.

Xiao, B.; Sanders, M.; Underwood, E.; Heath, R.; Mayer, F.; Carmena, D.; Jing, C.; Walker, P.; Eccleston, J.; Haire, L.; Saiu, P.; Howell, S.; Aasland, R.; Martin, S.; Carling, D. and Gamblin, S. 2011. Structure of mammalian AMPK and its regulation by ADP. Nature, 472: 230-233.

Yu, S.; Kim, J.; Chung, H.; Jung, K.; Lee, Y.; Yoon, D.; Lee, S.; Choi, I.; Bottema, C.; Sang, B. and Lee, J. 2005. Molecular cloning and characterization of bovine PRKAG3 gene: structure, expression and single nucleotide polymorphism detection. J Anim Breed Genetic, 122: 294-301.

Zuker, M. 2003. Mfold web server for nucleic acid folding and hybridization prediction. Nucleic Acids Res, 31: 3406-3415.

Archivos de zootecnia vol. 63, núm. 241, p. 132. 